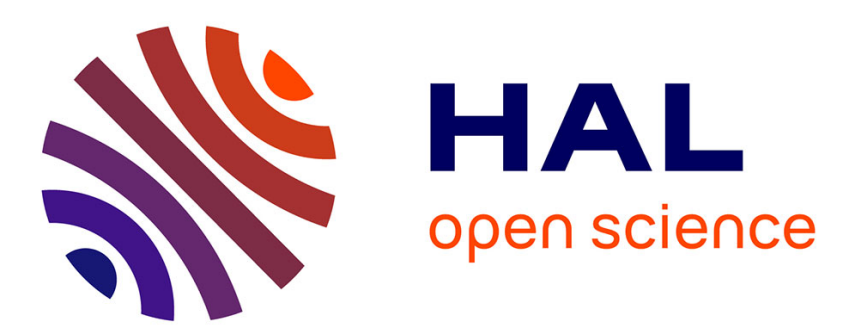

\title{
Two-Way Ambiguity in 2D Projective Reconstruction from Three Uncalibrated 1D Images \\ Long Quan
}

\section{To cite this version:}

Long Quan. Two-Way Ambiguity in 2D Projective Reconstruction from Three Uncalibrated 1D Images. IEEE Transactions on Pattern Analysis and Machine Intelligence, 2001, 23 (2), pp.212-216. 10.1109/34.908971 . inria-00590145

\section{HAL Id: inria-00590145 \\ https://hal.inria.fr/inria-00590145}

Submitted on 3 May 2011

HAL is a multi-disciplinary open access archive for the deposit and dissemination of scientific research documents, whether they are published or not. The documents may come from teaching and research institutions in France or abroad, or from public or private research centers.
L'archive ouverte pluridisciplinaire HAL, est destinée au dépôt et à la diffusion de documents scientifiques de niveau recherche, publiés ou non, émanant des établissements d'enseignement et de recherche français ou étrangers, des laboratoires publics ou privés. 


\title{
Two-Way Ambiguity in 2D Projective Reconstruction from Three Uncalibrated 1D Images
}

\author{
Long QUAN \\ CNRS-INRIA \\ 655 avenue de l'Europe \\ 38330 Montbonnot, France \\ Email: Long.Quan@inrialpes.fr
}

\begin{abstract}
We show that there is in general a two-way ambiguity for $2 \mathrm{D}$ projective reconstruction from three uncalibrated 1D views, independent of the number of point correspondences. The two distinct projective reconstructions are exactly related by a quadratic transformation with the three camera centers as fundamental points. Unique 2D reconstruction is possible only when the three camera centers are aligned. By Carlsson duality, there is a dual two-way ambiguity for 2D projective reconstruction from six point correspondences, independent of the number of $1 \mathrm{D}$ views. The theoretical results are demonstrated on numerical examples.
\end{abstract}

Key words: 1D camera, vision geometry, ambiguity, reconstruction. 


\section{Introduction}

A standard CCD camera is commonly modeled as a $2 \mathrm{D}$ projective device that perspectively projects a point in $\mathcal{P}^{3}$ (the projective space of dimension 3 ) to a point in $\mathcal{P}^{2}$. By analogy, we can consider what we call a $1 \mathrm{D}$ projective camera [15], which projects a point in $\mathcal{P}^{2}$ to a point in $\mathcal{P}^{1}$. Many imaging systems using laser beams, infra-red or ultra-sounds acting only within a single plane can be modeled as 1D projective cameras [2]. Moreover in some situations, the usual $2 \mathrm{D}$ camera model is also closely related to this $1 \mathrm{D}$ one. The first example is a $2 \mathrm{D}$ affine camera viewing 3D lines: the direction vectors of 3D and image lines correspond to each other via a $1 \mathrm{D}$ projective camera model $[15,13]$. Another example [8] is that a $2 \mathrm{D}$ camera undergoing a planar motion reduces to a $1 \mathrm{D}$ camera on the trifocal plane of the $2 \mathrm{D}$ cameras.

The geometry of multiple 1D views is completely characterised by its associated trifocal tensor which has interesting uniqueness and minimality properties that the $2 \mathrm{D}$ trifocal tensor $[19,7]$ does not have. However explicit 2D reconstruction from the $1 \mathrm{D}$ trifocal tensor is ambiguous [13]. In this paper, we prove that there is a two-way ambiguity for $2 \mathrm{D}$ projective reconstruction from three 1D images. The two distinct projective structures are related by a quadratic transformation, with the three camera centers as fundamental points. Unique reconstruction is possible only when the three camera centers are aligned. We will deal only with uncalibrated $1 \mathrm{D}$ cameras. For the calibrated $1 \mathrm{D}$ case motivated by laser-guided navigation, refer to $[1,2]$. A preliminary version of these results appeared in $[13,14]$.

The paper is organised as follows. In Section 2 and 3, we review the 1D projective camera, its trifocal tensor and $2 \mathrm{D}$ projective reconstruction. We then prove the major results in Section 4. The numerical simulation examples are given in Section 5 to support the theoretical developement. Finally, some concluding remarks in Section 6.

Throughout the paper, vectors are denoted in lower case boldface $\mathbf{x}, \mathbf{u} \ldots$, matrices and tensors in upper case boldface $\mathbf{A}, \mathbf{T} \ldots ;$ Scalars are any plain letters or lower case Greek $a, u, A, \lambda \ldots$ Some basic tensor notation is used: covariant indices are written as subscripts and contravariant indices as superscripts. e.g. the homogeneous coordinates of a point $\mathbf{x}$ in $\mathcal{P}^{2}$ are written with an upper index $\mathbf{x}=\left(x^{1}, x^{2}, x^{3}\right)^{T}$. A matrix $\mathbf{A}$ may also be written with two indices like $\mathbf{A}_{j}^{i}$, where $i$ indexes rows and $j$ columns. The implicit summation convention is also adopted. 


\section{1D projective camera and its trifocal tensor}

We first review the one-dimensional uncalibrated camera that was abstracted from the study of the geometry of lines under affine cameras $[15,13]$. We can also introduce it directly by analogy to a $2 \mathrm{D}$ projective camera.

A 1D projective camera projects a homogeneous point $\mathbf{x}=\left(x^{1}, x^{2}, x^{3}\right)^{T}$ in $\mathcal{P}^{2}$ (the projective plane) to a point $\mathbf{u}=\left(u^{1}, u^{2}\right)^{T}$ in $\mathcal{P}^{1}$ (the projective line). This projection may be described by a $2 \times 3$ homogeneous matrix $\mathbf{M}$ as follows:

$$
\lambda \mathbf{u}=\mathbf{M}_{2 \times 3} \mathbf{x} .
$$

Now consider the geometric matching constraints available for points seen in multiple 1D views, analogously to the $2 \mathrm{D}$ camera case $[17,18,9,4,5,3,19,7,12]$. It turns out that there is a constraint only in the case of 3 views. For 2 views, any two optical rays from the two 1D cameras must intersect in $2 \mathrm{D}$ space, so there can be no constraint.

Let the three views of the same point $\mathbf{x}$ be given as follows:

$$
\left\{\begin{aligned}
\lambda \mathbf{u} & =\mathbf{M} \mathbf{x} \\
\lambda^{\prime} \mathbf{u}^{\prime} & =\mathbf{M}^{\prime} \mathbf{x} \\
\lambda^{\prime \prime} \mathbf{u}^{\prime \prime} & =\mathbf{M}^{\prime \prime} \mathbf{x}
\end{aligned}\right.
$$

These equations can be rewritten in matrix form as

$$
\left(\begin{array}{cccc}
\mathbf{M} & \mathbf{u} & 0 & 0 \\
\mathbf{M}^{\prime} & 0 & \mathbf{u}^{\prime} & 0 \\
\mathbf{M}^{\prime \prime} & 0 & 0 & \mathbf{u}^{\prime \prime}
\end{array}\right)\left(\begin{array}{c}
\mathbf{x} \\
-\lambda \\
-\lambda^{\prime} \\
-\lambda^{\prime \prime}
\end{array}\right)=0 .
$$

The vector $\left(\mathbf{x},-\lambda,-\lambda^{\prime},-\lambda^{\prime \prime}\right)^{T}$ cannot be zero, so

$$
\left|\begin{array}{cccc}
\mathbf{M} & \mathbf{u} & 0 & 0 \\
\mathbf{M}^{\prime} & 0 & \mathbf{u}^{\prime} & 0 \\
\mathbf{M}^{\prime \prime} & 0 & 0 & \mathbf{u}^{\prime \prime}
\end{array}\right|=0
$$

Expansion of this determinant produces the unique trilinear constraint linking the three views 


$$
\mathbf{T}_{i j k} \mathbf{u}^{i} \mathbf{u}^{\prime j} \mathbf{u}^{\prime \prime k}=0
$$

where $\mathbf{T}_{i j k}$ is a $2 \times 2 \times 2$ homogeneous tensor - the trifocal tensor of the $1 \mathrm{D}$ cameras. The components $\mathbf{T}_{i j k}$ are $3 \times 3$ minors involving three views with each row from a different view of the $6 \times 3$ joint projection matrix $\left(\begin{array}{c}\mathbf{M} \\ \mathbf{M}^{\prime} \\ \mathbf{M}^{\prime \prime}\end{array}\right)$.

It is easy to see that any constraint obtained by adding further views reduces to a trilinearity, so no higher-order constraints exist. Moreover, the $2 \times 2 \times 2$ homogeneous tensor has $7=2 \times 2 \times 2-1$ d.o.f., so it is a minimal parametrization of three views in the uncalibrated setting since three views have exactly $3 \times(2 \times 3-1)-(3 \times 3-1)=7$ d.o.f. up to a projective transformation in $\mathcal{P}^{2}$.

In summary, the trifocal tensor of three 1D cameras encapsulates exactly the information needed for projective reconstruction in $\mathcal{P}^{2}$ : it is the unique matching constraint, it minimally parametrizes the three views and it can be estimated linearly. Contrast this to the $2 \mathrm{D}$ image case in which the multilinear constraints are algebraically redundant and linear estimation is only an approximation based on over-parametrization.

\section{Two way ambiguity of $2 \mathrm{D}$ projective reconstruction}

Projective reconstruction in $\mathcal{P}^{3}$ can be viewed as being equivalent to the rescaling of the image points in $\mathcal{P}^{2}$ according to [19]. For any single triplet of $1 \mathrm{D}$ image points in three views $(c f$. Equation (2)), the scale factors $\lambda, \lambda^{\prime}$ and $\lambda^{\prime \prime}$ are arbitrary scale factors, however, taken as a whole across many triplets, the scale factors encode the projective structure of the points $\mathbf{x}$ in $\mathcal{P}^{2}$. One way to explicitly recover the scale factors $\left(\lambda, \lambda^{\prime}, \lambda^{\prime \prime}\right)^{T}$ is to notice that the rescaled image coordinates $\left(\lambda \mathbf{u}, \lambda^{\prime} \mathbf{u}^{\prime}, \lambda^{\prime \prime} \mathbf{u}^{\prime \prime}\right)^{T}$ should lie in the joint image, or equivalently to observe the following matrix identity:

$$
\left(\begin{array}{cc}
\mathbf{M} & \lambda \mathbf{u} \\
\mathbf{M}^{\prime} & \lambda^{\prime} \mathbf{u}^{\prime} \\
\mathbf{M}^{\prime \prime} & \lambda^{\prime \prime} \mathbf{u}^{\prime \prime}
\end{array}\right)=\left(\begin{array}{c}
\mathbf{M} \\
\mathbf{M}^{\prime} \\
\mathbf{M}^{\prime \prime}
\end{array}\right)\left(\begin{array}{ll}
\mathbf{I}_{3 \times 3} & \mathbf{x}
\end{array}\right)
$$


The rank of the left matrix is therefore at most 3 , so all $4 \times 4$ minors vanish. Expanding by cofactors in the last column gives homogeneous linear equations in the components of $\lambda \mathbf{u}, \lambda^{\prime} \mathbf{u}^{\prime}$ and $\lambda^{\prime \prime} \mathbf{u}^{\prime \prime}$

$$
\left(\epsilon^{j l} \epsilon^{k m} \mathbf{T}_{i j k}\left(\lambda \mathbf{u}^{i}\right)\right)-\mathbf{e}_{1}^{\prime \prime}\left(\lambda^{\prime} \mathbf{u}^{\prime}\right)^{T}+\left(\lambda^{\prime \prime} \mathbf{u}^{\prime \prime}\right) \mathbf{e}_{1}^{\prime T}=\mathbf{0}_{2 \times 2},
$$

where all coefficients $T_{i j k}, \mathbf{e}_{1}^{\prime \prime}$ and $\mathbf{e}_{1}^{\prime}$ are $3 \times 3$ minors of the joint projection matrix $\left(\begin{array}{l}\mathbf{M} \\ \mathbf{M}^{\prime} \\ \mathbf{M}^{\prime \prime}\end{array}\right)$ and $\epsilon^{11}=\epsilon^{22}=0, \epsilon^{12}=1, \epsilon^{21}=-1$.

There are two types of $3 \times 3$ minors of the joint projection matrix: Those involving three views with one row from each view and those involving two views with two rows from one view and one from the other. The first type gives the 8 components of the tensor $\mathbf{T}_{2 \times 2 \times 2}$ and the second gives 12 components of the "epipoles" $\mathbf{e}_{1}, \mathbf{e}_{2}, \mathbf{e}_{1}^{\prime}, \mathbf{e}_{2}^{\prime}, \mathbf{e}_{1}^{\prime \prime}, \mathbf{e}_{2}^{\prime \prime}$. The epipoles are defined by analogy with the $2 \mathrm{D}$ camera case, as the projection of one camera center onto another view (see Figure 1).

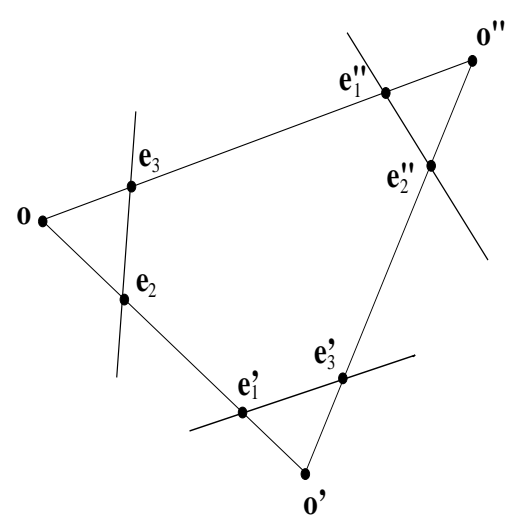

Figure 1: The geometry of three 1D images with the associated epipoles.

At present only $T_{i j k}$ has been estimated, the epipoles are still unknown. To find the rescaling factors for projective reconstruction, we need to solve for these epipoles. One way to proceed is as follows. Taking $\mathbf{x}$ to be the projection center of the second view $\mathbf{o}^{\prime}$, and projecting into the three views, Equation (6) reduces to

$$
\epsilon^{j l} \epsilon^{k m} \mathbf{T}_{i j k}\left(\lambda \mathbf{e}_{2}^{i}\right)-\mathbf{0}_{2 \times 2}+\left(\lambda^{\prime \prime} \mathbf{e}_{2}^{\prime \prime}\right) \mathbf{e}_{1}^{\prime T}=\mathbf{0}_{2 \times 2},
$$

i.e.

$$
\lambda \epsilon^{j l} \epsilon^{k m} \mathbf{T}_{i j k} \mathbf{e}_{2}^{i}=-\lambda^{\prime \prime} \mathbf{e}_{2}^{\prime \prime} \mathbf{e}_{1}^{T}
$$


As $\mathbf{e}_{1}^{\prime} \mathbf{e}^{\prime \prime T}$ has rank 1 , so does $\mathbf{T}_{i j k} \mathbf{e}_{2}^{i}$. Its $2 \times 2$ determinant must vanish, i.e.

$$
\operatorname{det}\left(\mathbf{T}_{i j k} \mathbf{e}_{2}^{i}\right)=0
$$

As each entry of the $2 \times 2$ matrix is homogeneous linear in $\mathbf{e}_{2}=(u, v)^{T}$, the expansion of $\operatorname{det}\left(\mathbf{T}_{i j k} \mathbf{e}_{2}^{i}\right)$ gives a homogeneous quadratic

$$
\alpha u^{2}+\beta u v+\gamma v^{2}=0
$$

where $\alpha, \beta, \gamma$ are known in terms of $\mathbf{T}_{i j k}$.

Doing the same thing with the projection center of the third view $\mathbf{o}^{\prime \prime}$ gives

$$
\lambda \epsilon^{j l} \epsilon^{k m} \mathbf{T}_{i j k} \mathbf{e}_{3}^{i}=\lambda^{\prime} \mathbf{e}_{1}^{\prime \prime} \mathbf{e}_{3}^{\prime T}
$$

and hence

$$
\operatorname{det}\left(\mathbf{T}_{i j k} \mathbf{e}_{3}^{i}\right)=0
$$

In other words, it leads to exactly the same quadratic equation (7) with $\mathbf{e}_{3}$ replacing $\mathbf{e}_{2}$. The two solutions of the quadratic (7) are $\mathbf{e}_{2}$ and $\mathbf{e}_{3}$ - only the ordering remains ambiguous.

The other epipoles are easily obtained, $\mathbf{e}_{1}^{\prime}$ and $\mathbf{e}_{3}^{\prime}$ by factorizing the matrix $\mathbf{T}_{i j k} \mathbf{e}_{1}^{\prime j}$ and $\mathbf{e}_{1}^{\prime \prime}$ and $\mathbf{e}_{2}^{\prime \prime}$ by factorizing $\mathbf{T}_{i j k} \mathbf{e}_{1}^{\prime \prime i}$. Only two of these $8=2 \times 2 \times 2$ ordering possibilities turn out to be internally self consistent, so finally we obtain two possible solutions for the 6 epipoles

$$
\left\{\mathbf{e}_{2}, \mathbf{e}_{3}\right\},\left\{\mathbf{e}_{1}^{\prime}, \mathbf{e}_{3}^{\prime}\right\},\left\{\mathbf{e}_{1}^{\prime \prime}, \mathbf{e}_{2}^{\prime \prime}\right\}
$$

and

$$
\left\{\mathbf{e}_{3}, \mathbf{e}_{2}\right\},\left\{\mathbf{e}_{3}^{\prime}, \mathbf{e}_{1}^{\prime}\right\},\left\{\mathbf{e}_{2}^{\prime \prime}, \mathbf{e}_{1}^{\prime \prime}\right\}
$$

Each solution for the epipoles gives a distinct projective reconstruction, so

Theorem 1 There are in general two distinct $2 D$ projective reconstructions from three $1 D$ views, independent of the number of point correspondences.

Another constructive proof of this result can be found in [15] based on direct determination of the projection matrix from the trifocal tensor instead of the re-scaling via epipoles used in this section. 


\section{Quadratic transformation between two distinct pro- jective reconstructions}

In this section, we prove that the two solutions for projective reconstruction are related by a quadratic transformation in $\mathcal{P}^{2}$.

Quadratic transformations are the simplest type of non-linear polynomial transformation between projective spaces, they are also called Cremona transformation [16]. Inversion with respect to a circle in the Euclidean plane is an example of a quadratic transformation. These tools have been used by Maybank [10] to study ambiguities of structure from motion [11, 6]. We will first briefly review some key properties of quadratic transformations necessary for our developement. See $[16,10]$ for more details on quadratic transformations.

\subsection{Quadratic transformations}

The correspondence set up by a quadratic transformation $\Phi: \mathbf{x} \rightarrow \mathbf{y}$ between two projective planes (x-plane and $\mathbf{y}$-plane) is one-one, except at three special points for which the defining quadratic polynomials $\phi_{i}, i=1,2,3$ all vanish, so that the transformed point is not uniquely defined. These three points are called the fundamental points of $\Phi$. Let the three fundamental points be $\mathbf{x}_{i}$ and $\mathbf{y}_{i}, i=1,2,3$ and assign them the canonical projective coordinates as

$$
\begin{aligned}
& \mathbf{x}_{1}=\mathbf{y}_{1}=(1,0,0)^{T}, \\
& \mathbf{x}_{2}=\mathbf{y}_{2}=(0,1,0)^{T}, \\
& \mathbf{x}_{3}=\mathbf{y}_{3}=(0,0,1)^{T} .
\end{aligned}
$$

The canonical quadratic transformation $\Phi_{0}$ is then given by

$$
\left(y^{1}, y^{2}, y^{3}\right)^{T}=\left(x^{2} x^{3}, x^{1} x^{3}, x^{1} x^{2}\right)^{T},
$$

or usually written as $\left(y^{1}, y^{2}, y^{3}\right)^{T}=\left(\frac{1}{x^{1}}, \frac{1}{x^{2}}, \frac{1}{x^{3}}\right)^{T}$.

The inverse $\Phi^{-1}=\Phi$ is therefore also a canonical quadratic transformation. The three lines through the fundamental points are called the fundamental lines and transform into the three fundamental points of $\Phi^{-1}$. Any other line through a fundamental point yields another line through the fundamental point corresponding to the 'opposite' fundamental line. A line that 
does not pass through any fundamental point transforms into a conic through the fundamental points. A conic through these three points transforms into a line. A general quadratic transformation $\Phi$ of the projective plane can be reduced to the standard quadratic transformation $\Phi_{0}$ by applying collineations in both the $\mathbf{x}$-plane and the $\mathbf{y}$-plane.

\subsection{Determination of reference points in $\mathcal{P}^{1}$ and $\mathcal{P}^{2}$}

To construct the quadratic transformation in an explicit manner, we need to choose a canonical projective basis in each $1 \mathrm{D}$ image and in the $2 \mathrm{D}$ plane. The two epipoles on each image line provide two natural reference points in $\mathcal{P}^{1}$ while the three camera centers provide three reference points in $\mathcal{P}^{2}$. We still lack unit points, both for $\mathcal{P}^{1}$ and $\mathcal{P}^{2}$. The choice of these points can be made as follows.

We may look for the set of invariant points for the three 1D images. Corresponding points $\mathbf{f} \leftrightarrow \mathbf{f}^{\prime} \leftrightarrow \mathbf{f}^{\prime \prime}$ are invariant if

$$
\lambda \mathbf{f}=\lambda^{\prime} \mathbf{f}^{\prime}=\lambda^{\prime \prime} \mathbf{f}^{\prime \prime}
$$

The triplet of corresponding points $\mathbf{f} \leftrightarrow \mathbf{f}^{\prime} \leftrightarrow \mathbf{f}^{\prime \prime}$ satisfies the trilinear constraint (5) as all corresponding points do, therefore,

$$
\mathbf{T}_{i j k} \mathbf{f}^{i} \mathbf{f}^{\prime j} \mathbf{f}^{\prime \prime k}=0
$$

i.e.

$$
\mathbf{T}_{i j k} \mathbf{f}^{i} \mathbf{f}^{j} \mathbf{f}^{k}=0
$$

This yields the following cubic equation in the unknown $\lambda=f^{1} / f^{2}$ :

$$
T_{111} \lambda^{3}+\left(T_{211}+T_{112}+T_{121}\right) \lambda^{2}+\left(T_{212}+T_{221}+T_{122}\right) \lambda+T_{222}=0
$$

As all of the coefficients of this cubic are real, it has in general either one or three real roots. This guarantees the existence of at least one real invariant point $\mathbf{r}$ in $\mathcal{P}^{2}$. This point can be used to determine a canonical basis in $\mathcal{P}^{2}$ and its invariant images $\mathbf{f}=\mathbf{f}^{\prime}=\mathbf{f}^{\prime \prime}$ in $\mathcal{P}^{1}$.

\subsection{Canonical basis in $\mathcal{P}^{1}$}

As we are working with uncalibrated images in a projective framework, without loss of generality we may apply a $2 \times 2$ homography to each image to bring its epipoles and fixed 
point to the canonical basis coordinates. Take the first set of solutions for the six epipoles $\left\{\mathbf{e}_{2}, \mathbf{e}_{3}\right\},\left\{\mathbf{e}_{1}^{\prime}, \mathbf{e}_{3}^{\prime}\right\},\left\{\mathbf{e}_{1}^{\prime \prime}, \mathbf{e}_{2}^{\prime \prime}\right\}$. We assume that homographies $\mathbf{A}, \mathbf{B}$ and $\mathbf{C}$ have been applied in three views such that

$$
\begin{array}{ll}
\mathbf{e}_{2}=(1,0)^{T}, & \mathbf{e}_{3}=(0,1)^{T}, \quad \mathbf{f}=(1,1)^{T} \\
\mathbf{e}_{1}^{\prime}=(1,0)^{T}, & \mathbf{e}_{3}^{\prime}=(0,1)^{T}, \quad \mathbf{f}^{\prime}=(1,1)^{T} \\
\mathbf{e}_{1}^{\prime \prime}=(1,0)^{T}, & \mathbf{e}_{2}^{\prime \prime}=(0,1)^{T}, \quad \mathbf{f}^{\prime \prime}=(1,1)^{T} .
\end{array}
$$

The first 1D image points are transformed into their canonical coordinates $(\theta, 1)^{T}=\mathbf{A}_{i}^{j} \mathbf{u}^{i}$ where $\theta$ is given by the cross-ratio

$$
\theta_{i}=\lambda_{i} / \mu_{i}=\left\{\mathbf{u}_{i}, \mathbf{f} ; \mathbf{e}_{2}, \mathbf{e}_{3}\right\}
$$

and

$$
\mathbf{u}_{i}=\lambda_{i} \mathbf{e}_{2}+\mu_{i} \mathbf{e}_{3}
$$

Similarly $\mathbf{u}^{\prime}$ and $\mathbf{u}^{\prime \prime}$ have canonical coordinates $\theta^{\prime}$ and $\theta^{\prime \prime}$ by $\mathbf{B}$ and $\mathbf{C}$.

The trifocal tensor expressed in these canonical bases is the canonical trifocal tensor $\mathbf{T}_{i j k}^{0}$ given by

$$
\mathbf{T}_{i j k}^{0}=\mathbf{T}_{a b c} \mathbf{A}_{i}^{a} \mathbf{B}_{j}^{b} \mathbf{C}_{k}^{c}
$$

\subsection{Canonical basis in $\mathcal{P}^{2}$}

Now in 2D space, we can equally choose a canonical basis defined by the three camera centers $\mathbf{o}, \mathbf{o}^{\prime}, \mathbf{o}^{\prime \prime}$ and the point $\mathbf{r}$ which has the invariant images $\mathbf{f}$ in all three views. Assume that a plane collineation be applied on the plane such that

$$
\mathbf{o}=(1,0,0)^{T}, \quad \mathbf{o}^{\prime}=(0,1,0)^{T}, \quad \mathbf{o}^{\prime \prime}=(0,0,1)^{T} \quad \text { and } \quad \mathbf{r}=(1,1,1)^{T}
$$

Obviously the plane collineation that brings to this canonical basis leaves the trifocal tensor $\mathbf{T}_{i j k}^{0}$ invariant as any plane collineation does.

\subsection{Quadratic transformation on the second projective structure}

The second set of solutions for the six epipoles $\left\{\mathbf{e}_{3}, \mathbf{e}_{2}\right\},\left\{\mathbf{e}_{3}^{\prime}, \mathbf{e}_{1}^{\prime}\right\},\left\{\mathbf{e}_{2}^{\prime \prime}, \mathbf{e}_{1}^{\prime \prime}\right\}$ is obtained by interchanging the pair of the epipoles of the first solution. Such a permutation of the epipoles gives 
a permutation of reference points on each image line, the canonical projective parametercanonical coordinate of the image point $\theta$-inverses as

$$
\theta^{\prime}=\left\{\mathbf{x}, \mathbf{f} ; \mathbf{e}_{1}, \mathbf{e}_{2}\right\}=1 /\left\{\mathbf{x}, \mathbf{f} ; \mathbf{e}_{2}, \mathbf{e}_{1}\right\}=1 / \theta
$$

This is equivalent to applying a homography

$$
\overline{\mathbf{I}}=\left(\begin{array}{ll}
0 & 1 \\
1 & 0
\end{array}\right)
$$

in each image. Such a homography transforms the canonical trifocal tensor $\mathbf{T}_{i j k}^{0}$ into $\mathbf{T}_{\bar{i} j \bar{k}}^{0}$ where ${ }^{-}$is the operator permuting the index 1,2 into 2 and 1 , as

$$
\mathbf{T}_{\overline{i j \bar{k}}}^{0}=\mathbf{T}_{a b c}^{0} \overline{\mathbf{I}}_{i}^{a} \overline{\mathbf{I}}_{j}^{b} \overline{\mathbf{I}}_{k}^{c}
$$

We see indeed that the second set of epipoles would have given a different tensor if no other things happened on the plane!

Now a quadratic transformation with the camera centers $\mathbf{o}, \mathbf{o}^{\prime}$ and $\mathbf{o}^{\prime \prime}$ as fundamental points can be applied to this reconstructed plane. This plane quadratic transformation has the consequence that any pencil of lines parameterized by $(\lambda, \mu)^{T}$ through a fundamental point tranforms homographically into the pencil with $(\mu, \lambda)^{T}$. The line of 1D image points can be viewed as a pencil of rays through the camera center, so the plane quadratic transformation induces a homographic transformation on the three image lines (which can be viewed, e.g., as the three fundamental lines of the quadratic transformation) of form

$$
\overline{\mathbf{I}}=\left(\begin{array}{ll}
0 & 1 \\
1 & 0
\end{array}\right) .
$$

This homographic transformation unpermutes the indices of the tensor, and hence undoes the first permutation created by interchanging the epipoles. The two steps combined leave the initial canonical trifocal tensor $T_{i j k}^{0}$ unchanged, as

$$
\mathbf{T}_{\overline{\bar{i} j \bar{k}}}^{0}=\mathbf{T}_{a b c}^{0} .
$$

In summary, the permutation of epipoles induced by selecting the second solution for the epipoles inverts the $1 \mathrm{D}$ image coordinates from $\theta$ to $1 / \theta$, so a $2 \mathrm{D}$ quadratic transformation is required to re-invert the $1 \mathrm{D}$ image coordinates back to $1 / 1 / \theta=\theta$. More formally:

Theorem 2 The two distinct projective reconstructions are related by a quadratic transformation in $\mathcal{P}^{2}$ with the three camera centers as fundamental points. 


\subsection{Unique reconstruction}

Above, we showed that there is in general a two-way ambiguity for projective reconstruction, with the two distinct reconstructions related by a quadratic transformation. However it is straightforward to see that if the homogeneous quadratic equation (7) has a double solution, we have $\mathbf{e}_{2}=\lambda \mathbf{e}_{3}$, i.e. the epipoles of the second and the third camera center coincide on the first image. This happens when the three camera centers are aligned, and in this case

Theorem 3 A unique reconstruction exists only when the three camera centers are aligned.

The uniqueness can be understood as a consequence of the fact that the quadratic transformation is not defined for collinear fundamental points.

\subsection{Dualization}

Recall that Carlsson duality $[4,5]$ for 2D cameras states that a projective reconstruction problem in $\mathcal{P}^{3}$ with $n 2 \mathrm{D}$ point correspondences and $m 2 \mathrm{D}$ images is equivalent (' $d u a l$ ') to one with $m+4$ points and $n-4$ images. Adapting this duality to 1D cameras, the problem with $n$ points and $m$ images is dual to the one with $m+3$ points and $n-3$ images. Dualizing the three $1 \mathrm{D}$ view problem gives

Theorem 4 There is in general a two-way ambiguity for projective reconstruction from six points independent of the number of views.

\section{$5 \quad$ Numerical simulations}

The theoretical results developed in this paper were tested using numerical simulations. We took a regular grid as illustrated in Figure 2, then simulated a 1D camera taking three images of this grid in three different positions. The two 2D projective reconstructions are illustrated in Figure 3. We can see that the first solution is indeed a projective transformation of the original grid, while the second is quadratically transformed: collinear points are transformed into points lying on conic sections.

In another experiment, we placed the three camera centers along a line. The resulting reconstruction confirms the uniqueness of the solution as illustrated in Figure 4. 


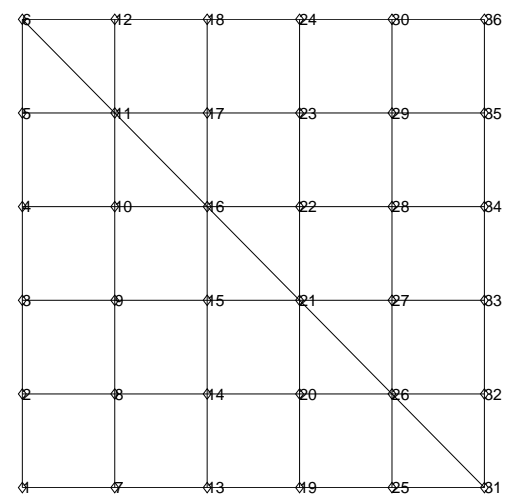

Figure 2: The original planar regular grid.

a.

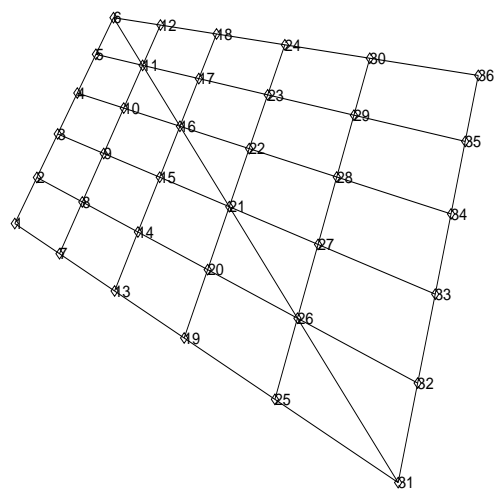

b.

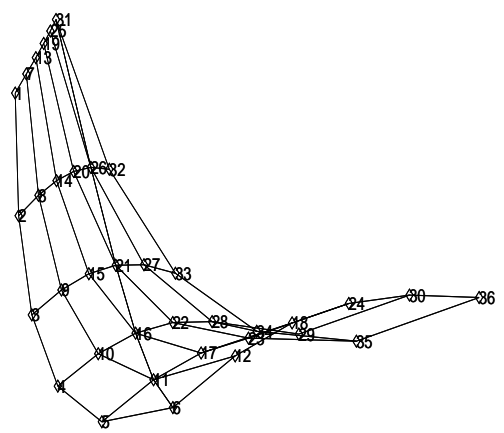

Figure 3: a. The first 2D projective reconstruction of the grid. b. The second 2D projective reconstruction which is deformed by a quadratic transformation.

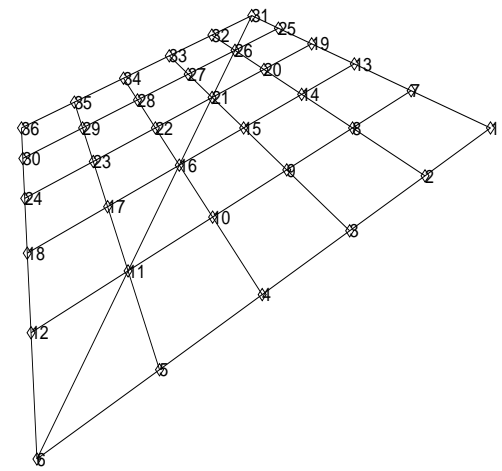

Figure 4: The unique 2D projective reconstruction of the grid when the three camera centers are aligned. 


\section{Discussion}

We have shown that there is in general a two way ambiguity for $2 \mathrm{D}$ projective reconstruction from three uncalibrated 1D images, independent of the number of point correspondences. By Carlsson duality, there is also a two-way ambiguity from 6 point correspondences, independent of the number of $1 \mathrm{D}$ images. More precisely, we showed that the two distinct projective reconstructions are related by a quadratic transformation with the three camera centers as fundamental points. A unique reconstruction exists when the three camera centers are aligned. These theoretical results were also validated on numerical simulations. These results give a new insight into the intrinsic structure of projective reconstruction, and may provide interesting hints for the study of the multiple solutions of $3 \mathrm{D}$ projective reconstruction from $2 \mathrm{D}$ cameras.

\section{Acknowledgement}

This work was partly supported by European project CUMULI which is gratefully acknowledged. Many key ideas developed in this paper come from discussions with S. Maybank, B. Triggs, S. Carlsson, R. Mohr and O. Faugeras. S. Maybank initially suggested the possibility of quadratic transformations and S. Carlsson suggested the dual form of the ambiguity.

\section{References}

[1] K. Åström. Invariance Methods for Points, Curves and Surfaces in Computational Vision. PhD thesis, Lund University, 1996.

[2] K. Åström and M. Oskarsson. Solutions and ambiguities of the structure and motion problem for 1d retinal vision. Journal of Mathematical Imaging and Vision, 12:121-135, 2000 .

[3] T. Buchanan. The twisted cubic and camera calibration. Computer Vision, Graphics and Image Processing, 42(1):130-132, April 1988. 
[4] S. Carlsson. Duality of reconstruction and positioning from projective views. In Workshop on Representation of Visual Scenes, Cambridge, Massachusetts, USA, pages 85-92, June 1995.

[5] S. Carlsson and D. Weinshall. Dual computation of projective shape and camera positions from multiple images. International Journal of Computer Vision, 27(3):227-241, May 1998.

[6] O. Faugeras and S. Maybank. Motion from point matches: Multiplicity of solutions. International Journal of Computer Vision, 3(4):225-246, 1990.

[7] O. Faugeras and B. Mourrain. On the geometry and algebra of the point and line correspondences between $n$ images. In Proceedings of the 5th International Conference on Computer Vision, Cambridge, Massachusetts, USA, pages 951-956, June 1995.

[8] O. Faugeras, L. Quan, and P. Sturm. Self-calibration of a 1d projective camera and its application to the self-calibration of a $2 \mathrm{~d}$ projective camera. In Proceedings of the 5th European Conference on Computer Vision, Freiburg, Germany, pages 36-52, June 1998.

[9] R.I. Hartley. A linear method for reconstruction from lines and points. In E. Grimson, editor, Proceedings of the 5th International Conference on Computer Vision, Cambridge, Massachusetts, USA, pages 882-887. IEEE, IEEE Computer Society Press, June 1995.

[10] S. Maybank. Theory of Reconstruction from Image Motion. Springer-Verlag, 1993.

[11] S.J. Maybank and A. Shashua. Ambiguity in reconstruction from images of six points. In Proceedings of the 6th International Conference on Computer Vision, Bombay, India, pages 703-708, 1998.

[12] L. Quan. Invariants of six points and projective reconstruction from three uncalibrated images. IEEE Transactions on Pattern Analysis and Machine Intelligence, 17(1):34-46, January 1995.

[13] L. Quan. Uncalibrated 1D projective camera and 3D affine reconstruction of lines. In Proceedings of the Conference on Computer Vision and Pattern Recognition, Puerto Rico, USA, pages 60-65, June 1997. 
[14] L. Quan. Inherent two-way ambiguity in 2d projective reconstruction from three uncalibrated 1d images. In Proceedings of the 7th International Conference on Computer Vision, Corfu, Greece, pages 344-349, 1999.

[15] L. Quan and T. Kanade. Affine structure from line correspondences with uncalibrated affine cameras. IEEE Transactions on Pattern Analysis and Machine Intelligence, 19(8):834-845, August 1997.

[16] J.G. Semple and G.T. Kneebone. Algebraic Projective Geometry. Oxford Science Publication, 1952.

[17] A. Shashua. Algebraic functions for recognition. IEEE Transactions on Pattern Analysis and Machine Intelligence, 17(8):779-789, August 1995.

[18] M. Spetsakis and J. Aloimonos. A unified theory of structure from motion. In Proceedings of DARPA Image Understanding Workshop, pages 271-283, 1990.

[19] B. Triggs. Matching constraints and the joint image. In E. Grimson, editor, Proceedings of the 5th International Conference on Computer Vision, Cambridge, Massachusetts, USA, pages 338-343. IEEE, IEEE Computer Society Press, June 1995. 